\title{
BMJ Open Estimated reductions in cardiovascular and gastric cancer disease burden through salt policies in England: an IMPACT $\mathrm{NCD}_{\mathrm{N}}$ microsimulation study
}

Chris Kypridemos, ${ }^{1}$ Maria Guzman-Castillo, ${ }^{1}$ Lirije Hyseni, ${ }^{1}$ Graeme L Hickey, ${ }^{2}$ Piotr Bandosz, ${ }^{1,3}$ lain Buchan, ${ }^{4}$ Simon Capewell, ${ }^{1}$ Martin O'Flaherty ${ }^{1}$

To cite: Kypridemos C, Guzman-Castillo M, Hyseni L, et al. Estimated reductions in cardiovascular and gastric cancer disease burden through salt policies in England: an IMPACT ${ }_{N C D}$ microsimulation study. BMJ Open 2017:7:e013791. doi:10.1136/bmjopen-2016013791

- Prepublication history and additional material is available. To view please visit the journal (http://dx.doi.org/ 10.1136/bmjopen-2016013791).

Received 7 August 2016 Revised 17 November 2016 Accepted 6 January 2017

CrossMark

${ }^{1}$ Department of Public Health and Policy, University of Liverpool, Liverpool, UK ${ }^{2}$ Department of Biostatistics, University of Liverpool, Liverpool, UK

${ }^{3}$ Department of Prevention and Medical Education, Medical University of Gdansk, Gdansk, Poland

${ }^{4}$ Farr Institute @ HeRC, University of Manchester, Manchester, UK

Correspondence to Dr Chris Kypridemos; ckyprid@liverpool.ac.uk

\section{ABSTRACT}

Objective: To estimate the impact and equity of existing and potential UK salt reduction policies on primary prevention of cardiovascular disease (CVD) and gastric cancer (GCa) in England.

Design: A microsimulation study of a close-to-reality synthetic population. In the first period, 2003-2015, we compared the impact of current policy against a counterfactual 'no intervention' scenario, which assumed salt consumption persisted at 2003 levels. For 2016-2030, we assumed additional legislative policies could achieve a steeper salt decline and we compared this against the counterfactual scenario that the downward trend in salt consumption observed between 2001 and 2011 would continue up to 2030 .

Setting: Synthetic population with similar characteristics to the non-institutionalised population of England.

Participants: Synthetic individuals with traits informed by the Health Survey for England.

Main measure: CVD and GCa cases and deaths prevented or postponed, stratified by fifths of socioeconomic status using the Index of Multiple Deprivation.

Results: Since 2003, current salt policies have prevented or postponed $\sim 52000$ CVD cases (IQR: 34 000-76 000) and 10000 CVD deaths (IQR: 300017000 ). In addition, the current policies have prevented 5000 new cases of GCa (IQR: 2000-7000) resulting in about 2000 fewer deaths (IQR: 0-4000). This policy did not reduce socioeconomic inequalities in CVD, and likely increased inequalities in GCa. Additional legislative policies from 2016 could further prevent or postpone 19000 CVD cases (IQR: 8000$30000)$ and 3600 deaths by 2030 (IQR: $-400-8100)$ and may reduce inequalities. Similarly for GCa, 1200 cases (IQR: $-200-3000)$ and 700 deaths (IQR: $-900-$ 2300) could be prevented or postponed with a neutral impact on inequalities.

Conclusions: Current salt reduction policies are powerfully effective in reducing the CVD and GCa burdens overall but fail to reduce the inequalities involved. Additional structural policies could achieve further, more equitable health benefits.

\section{Strengths and limitations of this study}

- Our study uses a technically advanced dynamic microsimulation model that synthesises information from the best available sources of information on population exposures to salt, and other non-communicable disease-related risk factor.

- Many assumptions must be made with such models; yet, in spite of the potential frailty of such assumptions this model validated well against observed cardiovascular disease and gastric cancer incidence and mortality in real populations, even when multiply stratified.

- The main assumption for the evaluation of current policy was that the decline in salt consumption observed since 2003 was fully attributable to the implemented policy.

- We could not find a sufficiently large data set with individual-level 24-hour urine sodium measurements and other non-communicable disease-related risk factor information. Therefore, we developed a stochastic process to overcome this and synthesise information from multiple sources, which increased the overall uncertainty of the model and is reflected in our reported uncertainty estimates.

- To ensure transparency, we have made IMPACT $_{\text {NCD }}$ source code open under GNU GPLv3 licence.

\section{BACKGROUND}

Excess salt consumption is associated with higher risk of cardiovascular disease (CVD) and gastric cancer (GCa). ${ }^{1}{ }^{2}$ Globally, more than 1.5 million CVD-related deaths every year can be attributed to the excess salt intake. $^{3}$ Further salt-related deaths come from GCa. Health policies worldwide, therefore, aim to reduce dietary salt intake. ${ }^{4}$ Furthermore, the WHO recommends reducing population exposure to salt as one 
of the 'best buy' strategies to prevent non-communicable diseases, highlighting its cost-effectiveness and feasibility. ${ }^{5}$

Since 2003, the UK has had one of the world's most successful salt reduction strategies, including public awareness campaigns, food labelling and 'voluntary' reformulation of processed foods. ${ }^{6}$ The strategy components and the evolution of the strategy over the years have been described in detail elsewhere. ${ }^{8}$ This package of measures is regularly evaluated and has been monitored through nationally representative surveys using 24-hour urine collection measurements. ${ }^{9}$ Between 2001 and 2011, the mean salt consumption in the UK dropped from 9.5 to $8.1 \mathrm{~g} /$ day $^{10}$ - a success, however, still far from the national target of $6 \mathrm{~g} /$ day. ${ }^{11}$

In the UK, salt consumption is higher in more deprived groups. ${ }^{12}{ }^{13}$ Therefore, interventions aiming to reduce salt consumption should ideally aim to also reduce socioeconomic inequalities in health. Unfortunately, the current UK strategy might potentially increase socioeconomic inequality because awareness campaigns, food labelling and voluntary reformulation can be more effective among the more health conscious, affluent individuals. ${ }^{14-17}$ Indeed, evidence suggests the socioeconomic gradient in salt consumption might have worsened during the programme. ${ }^{13} 18$ In contrast, modelling studies consistently suggest that more structural interventions can be more effective, cost-effective and equitable than the current UK policy. ${ }^{19}{ }^{20}$

Structural salt reduction policies are usually based on legislative initiatives like a mandatory reformulation of processed foods or taxation of high-salt foods. Such policies have already been adopted successfully in Argentina, South Africa, Portugal, Hungary and elsewhere, emphasising their feasibility. ${ }^{4}$ In fact, the actual number of countries currently implementing legislative measures has substantially increased since 2010, indicating a global move towards stricter salt reduction policies. $^{4}$

The aim of this study was to estimate the impact and equity of current UK salt reduction policy on CVD and GCa burden since 2003. We further compared current policy with other feasible policies to estimate possible additional incidence and mortality reductions.

\section{METHODS}

We used IMPACT $\mathrm{NCD}_{\mathrm{NC}}$, a discrete time, dynamic, stochastic microsimulation model to simulate the effect of current policy and compare it to counterfactual scenarios. We split our analysis into two periods. The first corresponds to years 2003-2015, for which we compared the potential benefits of current policies against a null intervention scenario. For the second period, 20162030, we explored the potential benefits of additional structural salt reduction policies, assuming they might lead to steeper declines in salt intake.

\section{Model description}

IMPACT $_{\mathrm{NCD}}$ simulates synthetic individuals and allows for greater flexibility and more detailed simulation, including different lag times between exposures and outcomes, socioeconomic gradients in trends of risk factors and a competing risk framework-a computationally intensive task for which we employed the Farr Institute's statistical high-performance computing facilities. ${ }^{21}$

The model synthesises information from Office for National Statistics (ONS) regarding English population structure by age, sex and socioeconomic status and the Health Survey for England ${ }^{22}$ regarding exposure to CVD and GCa-associated risk factors (see below) to generate a close-to-reality synthetic population. ${ }^{23}$ Well-established causal pathways between associated risk factors and disease are used to translate exposure into CVD and GCa incidence and mortality, in a competing risk framework. Effect sizes were taken from published meta-analyses and longitudinal studies (see online supplementary table S1). For salt, we assumed a mediated effect through systolic blood pressure on CVD incidence with 5-year mean lag time, and a direct effect on GCa incidence with a mean lag time of 8 years.

Outputs include CVD and GCa incidence and mortality in the synthetic population under different scenarios. A detailed description of IMPACT $\mathrm{NCD}_{\mathrm{NCD}}$ is provided in the online supplementary Chapters S2-S4.

\section{Risk factor modelling}

The exposure of the synthetic population to salt was informed by four nationally representative surveys employing 24-hour urine collections between 2001 and 2011. ${ }^{10} 24-26$ We used a stochastic process to enhance the information from these surveys with information from spot urine measurements (see detailed description in the online supplement Paragraph S3.3.2). Then, we used quantile regression to project daily salt consumption to 2030. Changes in salt consumption were transformed to systolic blood pressure changes using the metaregression equation of a meta-analysis of 103 trials. ${ }^{3}$ The ideal level of salt consumption is not clear (see appendix Text S4 in Mozaffarian et al). ${ }^{3}$ We allowed the level of ideal salt consumption under which no risk exist to vary between 1.5 and $6 \mathrm{~g} /$ day with a mode of $3.8 \mathrm{~g} /$ day, following a PERT distribution. ${ }^{27}$

Trends of other CVD and GCa-associated risk factors were also considered in this study by projecting the observed in Health Survey for England trends since 2001, up to 2030. For CVD, body mass index, total plasma cholesterol, diabetes mellitus (diagnosis or elevated glycated haemoglobin/no diabetes), smoking status (current/ex/never smoker), environmental tobacco exposure (binary variable), fruit and vegetable (portions/day) consumption, and physical activity (days with at least $30 \mathrm{~min}$ of moderate or vigorous physical activity/week) were included. Smoking duration, body mass index, and less than two portions of fruit and vegetable consumption were considered for $\mathrm{GCa} .{ }^{28}$ 
CVD was defined as the sum of coronary heart disease (CHD) and stroke (any type) cases. This study focuses on primary prevention; hence, only the first episode of CHD, stroke and GCa was considered. The competing risk framework allows individuals to develop CHD, stroke or GCa independently, and die from these or any other cause.

\section{Model outputs}

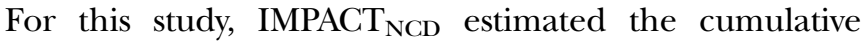
cases prevented or postponed and deaths prevented or postponed for the relevant period and for ages 30-84. The results were stratified by quintile groups of Index of Multiple Deprivation (QIMD), a relative measure of area deprivation widely used in England. ${ }^{29}$ Inspired by the slope index of inequality, ${ }^{30}$ we used two regression-based metrics, the 'absolute equity slope index' and the 'relative equity slope index', as equity measures of a policy. The former measures the impact of an intervention on absolute inequality; for instance, a value of 100 means 100 more cases were prevented or postponed in most deprived compared with least deprived areas, and absolute inequality was decreased. The latter takes into account pre-existing socioeconomic gradient of disease burden and measures the impact of an intervention on relative inequality; positive values mean the policy tackles relative inequality and negative that the policy generates relative inequality.

Owing to the assumed lag times, any changes in salt exposure in the 2003 to 2015 period will reflect on CVD incidence and mortality in years 2008 to 2020, and GCa incidence and mortality in years 2011-2023. Similarly, for the period 2016-2030, these changes will be reflected in CVD burden in 2021-2035 and in GCa burden in 2024-2038.

\section{Uncertainty analysis}

A probabilistic sensitivity analysis is incorporated in our estimates, as IMPACT $_{\mathrm{NCD}}$ implements a second-order Monte Carlo approach that allows the estimated uncertainty of model inputs to be propagated to the outputs. ${ }^{31}$ We summarise the output distributions by reporting medians and IQRs in the form of first and third quartiles. We also report the probability (Ps) that a policy scenario aspect is superior to the counterfactual one. For example, ' 100 cases prevented or postponed $(\mathrm{Ps}=80 \%)$ in scenario $\mathrm{A}$ ' is interpreted as 'in $80 \%$ of Monte Carlo iterations, at least one case has been prevented or postponed in scenario "A" comparing to the counterfactual scenario'. Consequently, in the remaining $20 \%$ of iterations, cases in scenario ' $\mathrm{A}$ ' were more than in the counterfactual scenario. This does not mean that scenario 'A' was harmful, but that its effect in those particular settings was not large enough to exceed the 'noise level' from other sources of uncertainty in the model. For a detailed description of the sources of uncertainty that were considered, please refer to the online supplement Chapter S6.

\section{Period 2003-2015 scenarios}

Two scenarios were simulated. The 'no intervention' scenario assumes that no salt-related interventions were implemented since 2003. Therefore, the salt exposure remained stable at the estimated level of 2003 for the period up to 2015. The 'current policy' scenario simulates the decline in salt consumption that was observed between 2003 and 2011, and projects it up to 2015, assuming a logarithmic decline.

\section{Period 2016-2030 scenarios}

Here, we modelled the potential effect of structural, legislative policies on salt intake, aimed to achieve feasible and ideal targets. First, we modelled a ccurrent policy' (baseline) scenario where the logarithmic decline observed from 2003 to 2011 was projected up to 2030.

In a 'feasible' target scenario, we assumed that in 2016, policies like mandatory reformulation and/or taxation of high-salt foods were implemented and as a result, the mean salt consumption will gradually decline to the national target of $6 \mathrm{~g} /$ day by 2020 for ages 19 to 64. Owing to lack of empirical evidence regarding the magnitude of the impact of such policies on salt, we allowed their target to vary between 5.8 and $7 \mathrm{~g}$ /day following a PERT distribution. The intervention was modelled to be more effective for individuals with higher salt consumption.

In an 'ideal' target scenario, we assumed mean salt intake to reach the ideal salt intake $3.8 \mathrm{~g}$ /day by 2025 for ages 19-64. The ideal salt consumption was modelled to vary between 1.5 and $6 \mathrm{~g}$ /day following a PERT distribution. Similarly to the previous scenario, the intervention was modelled to be more effective for individuals with higher salt consumption. The modelled trends of salt consumption for all scenarios are depicted in figure 1 .

\section{Other assumptions}

We assumed that CVD and GCa case fatality is improving by $5 \%$ and $2 \%$ annually, respectively, but the rate of improvement diminishes by $1 \%$ (relative) every year. Moreover, we assumed that there is a constant fatality rate socioeconomic gradient of $\sim 5 \%$ by QIMD level (halved for ages over 70) forcing the more deprived to experience worse disease outcomes. These assumptions are based on empirical evidence. ${ }^{32-35}$ Table 1 presents the key modelling assumptions.

\section{RESULTS}

We present our results separately for the two distinct periods, then an external validation of IMPACT $_{\mathrm{NCD}}$.

\section{Evaluation of current policy (2003-2015)}

Under the 'current policy' scenario, median salt consumption was reduced from 8.9 (IQR: 8.7-9.2) $\mathrm{g}$ /day in 2003 to 7.1 (IQR: 6.9-7.2) g/day in 2015. Socioeconomic 
inequalities in salt consumption remained and might even have increased as a result of the current policy.

Under the 'no intervention' scenario, IMPACT $\mathrm{NCD}_{\mathrm{NC}}$ estimated $\sim 1.3$ (IQR: 1.2-1.4) million new cases of CVD and 700000 (IQR: $680000-720000$ ) deaths from CVD. Likewise, the model estimated 68 000 (IQR: 61000 74000 ) new GCa cases and 41000 (IQR: 37 000-44 000) deaths.

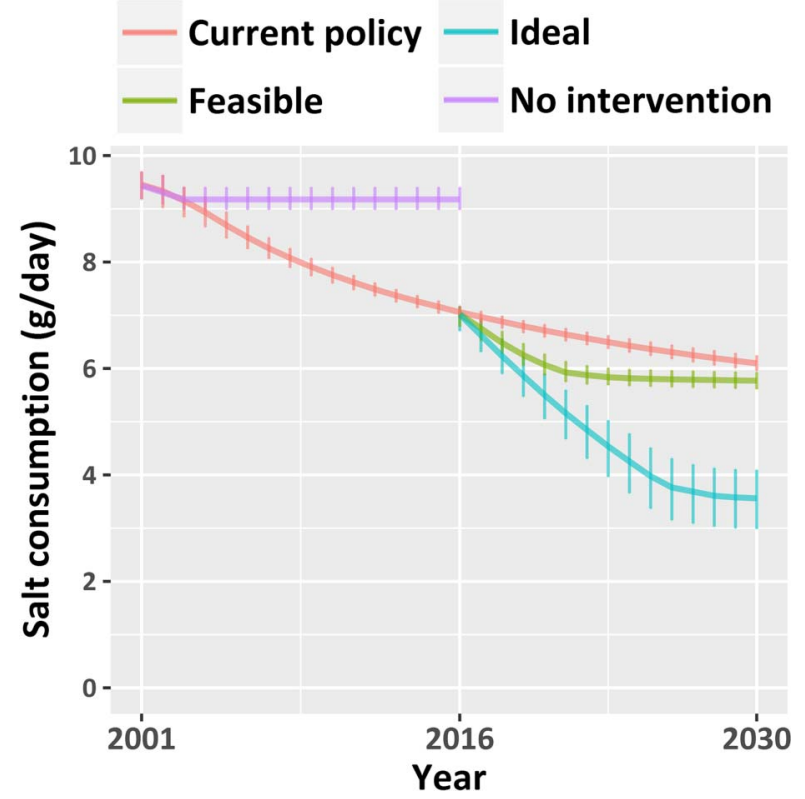

Figure 1 Modelled trends of median salt consumption in English population aged 30-84 under the four simulated scenarios. Error bars represent IQRs.
Compared with the 'no intervention' scenario, the salt reduction strategy resulted in about 52000 (IQR: 34 000-76 000; Ps=99\%) fewer new CVD cases, and 10000 (IQR: 3000-17 000; Ps=86\%) fewer CVD deaths. In addition, the current policy prevented around 5000 (IQR: 2000-7000; Ps=92\%) new cases of GCa resulting in 2000 (IQR: 0-4000; Ps=78\%) fewer GCA deaths.

When equity was considered, we estimated that the current policy has a rather neutral effect on tackling socioeconomic inequalities in CVD. The effect on GCa equity was more complex. Current policy apparently prevented or postponed fewer GCa cases in more deprived areas. However, GCa incidence increases with age and more affluent individuals tend to live longer. After directly standardising age and sex, the effect was essentially disappeared for absolute inequality bur remained for relative inequality (table 2 ).

\section{Future options (2016-2030)}

Under the 'current policy' scenario, IMPACT $_{\mathrm{NCD}}$ projected that median salt consumption would reduce further from 7.0 (IQR: $6.8-7.7$ ) $\mathrm{g} /$ day in 2016 to 6.2 (IQR: 5.9-6.2) g/day in 2030. The addition of structural policies might reach the national target of $6 \mathrm{~g}$ /day by 2020. The less feasible 'ideal' policy scenario was estimated to reach 3.6 (IQR: 3.0-4.1) g/day by 2030 . Inequality in salt consumption persisted under the 'current policy' projections and decreased moderately with the addition of structural policies.

Under the 'current policy' scenario, we calculated $~ 1.4$ million new cases of CVD (IQR: 1.3-1.4 million) and 530000 deaths (IQR: $510000-560000$ ). Similarly, for GCa we estimated some 80000 new cases (IQR: 65000

\section{Table $1 \quad$ IMPACT $_{\text {NCD }}$ key assumptions}

\begin{tabular}{ll}
\hline Population module & Migration is not considered. \\
& Social mobility is not considered. \\
QIMD is a marker of relative area deprivation with several versions since 2003. We considered all \\
version of QIMD identical. \\
We assume all salt that is consumed is excreted from urine and all urine sodium comes from salt \\
consumption. \\
We assume that the surveys used are truly representative of the population. \\
We assume that the decline in salt consumption observed since 2003 was fully attributable to the \\
implemented policy. \\
We assume multiplicative risk effects. \\
We assume log-linear dose-response for the continuous risk factors. \\
We assume that the effects of the risk factors on incidence and mortality are equal and risk factors are \\
not modifying survival. \\
We assume 5-year mean lag time for CVD and 8-year for GCa (except for the cumulative effect of \\
smoking on GCa where lag was assumed similar to CVD one). \\
We assume 100\% risk reversibility. \\
We assume that trends in disease incidence are attributable only to trends of the relevant modelled risk \\
factors. \\
Only well-accepted associations between upstream and downstream risk factors that have been \\
observed in longitudinal studies are considered. However, the magnitudes of the associations are \\
extracted from a series of nationally representative cross-sectional surveys (Health Survey for England). \\
For GCa, we assume that survival of 10 years after diagnosis equals remission.
\end{tabular}




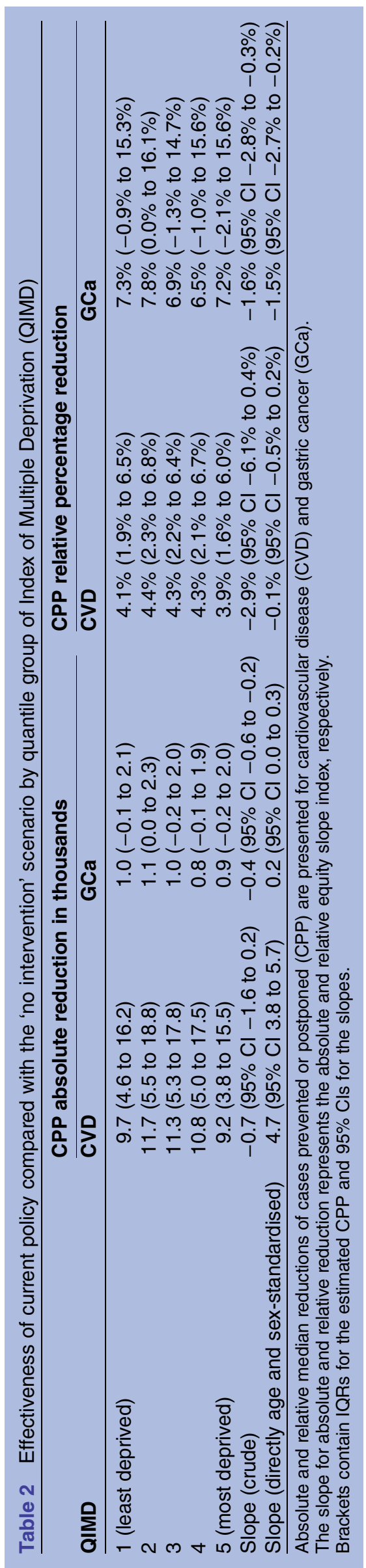

-93 000) and 42000 deaths (IQR: $35000-49000$ ). Approximately 20000 more cases of CVD and GCa can be prevented or postponed from the implementation of structural policies. Table 3 presents IMPACT $\mathrm{NCD}_{\mathrm{N}}$ estimates for the two counterfactual scenarios.

The addition of structural policies was more effective among the most deprived groups especially for CVD and might potentially decrease absolute socioeconomic inequality (table 4). As anticipated, the 'ideal' scenario had the largest impact on burden and inequality (table 5).

\section{Validation}

We assessed the eternal validity of the IMPACT $_{\mathrm{NCD}}$ model by comparing the estimated number of deaths from CVD and GCa against the observed number of deaths from the same causes for years 2006 to 2013 in England (figure 2). Detailed graphs by age group, sex, QIMD and disease can be found in the online supplement chapter S8. Overall, IMPACT $\mathrm{NCD}_{\mathrm{NCD}}$ is strongly validated even when mortality was highly stratified.

\section{DISCUSSION}

This is the first study to quantify the impact of UK salt reduction policies on CVD and GCa by socioeconomic group. We estimated that the current UK salt strategy has potentially prevented or postponed some 57000 new cases and 12000 deaths from CVD and GCa in England. The addition of structural policies and achievement on the national target by 2020 could potentially prevent or postpone a further 20000 new cases and 4000 deaths, while the 'ideal' combination of salt reduction policies might potentially prevent or postpone some 80000 new cases and 14000 deaths from CVD and GCa.

When equity is considered, the impact of the implemented strategy is more complex. Our results agree with previous studies ${ }^{13} 18$ that the socioeconomic gradient in salt consumption would not be reduced by these strategies. IMPACT $\mathrm{NCD}_{\mathrm{NC}}$ estimated that current policies might have a rather neutral impact of CVD socioeconomic inequalities (absolute and relative) and worsen GCa inequalities reflecting an older age distribution in more affluent groups. However, the addition of structural policies may reduce absolute socioeconomic inequality in CVD incidence and neutralise the negative impact of current policies on GCa inequalities.

Simpler modelling studies have previously examined the impact of a theoretical decrease in UK salt consumption. A $3 \mathrm{~g} /$ day reduction in salt consumption might prevent about 32000 CVD cases and 4500 CVD deaths in England and Wales in a 10-year period according to Barton et al, ${ }^{37}$ or 200000 fewer CVD events and 90000 fewer CVD deaths according to Dodhia $e t a l^{38}$ or almost 100000 fewer CVD deaths in 20 years according to Hedriksen et $a .^{39}$ Our results appear to echo the more conservative estimates by Barton et al. ${ }^{37}$ In addition, 
Table 3 Additional cases and deaths that can be potentially prevented or postponed (CPP, DPP) from the addition of structural policies to current policy, and under the 'ideal scenario'

\begin{tabular}{|c|c|c|c|c|}
\hline \multirow[b]{2}{*}{ Scenario } & \multicolumn{2}{|l|}{ Cardiovascular disease } & \multicolumn{2}{|l|}{ Gastric cancer } \\
\hline & CPP in thousands & DPP in thousands & CPP in thousands & DPP in thousands \\
\hline Feasible & 18.7 (8.0 to $29.5 ; \mathrm{Ps}=90 \%)$ & $3.6(-0.4$ to $8.1 ; P S=72 \%)$ & $1.2(-0.2$ to $3.0 ; P s=72 \%)$ & 0.7 ( -0.9 to $2.3 ; P s=63 \%)$ \\
\hline Ideal & $73.2(53.9$ to $94.3 ; P s=100 \%)$ & $11.0(6.5$ to $16.1 ; P s=95 \%)$ & 6.3 (3.4 to $9.6 ; P s=94 \%)$ & $3.1(1.1$ to $5.1 ; P s=86 \%)$ \\
\hline
\end{tabular}

Gillespie $e t a t^{20}$ model that was informed by experts' opinion to model policy effectiveness and equity estimated that mandatory salt reformulation might reduce socioeconomic inequalities in CHD. We reached reassuringly similar conclusions using a very different methodology.

Going further than previous studies, we modelled structural interventions and as being more effective for those individuals with the highest salt intakes. In the UK, about $70 \%$ of dietary salt comes from processed food. ${ }^{11}$ Since structural policies target processed foods, their effect would be stronger among those with higher consumption of processed food, and hence higher salt intake.

Some researchers claim that salt consumption lower than $7.5 \mathrm{~g}$ can actually increase the risk of CVD and overall mortality. ${ }^{40}{ }^{41}$ However, it appears that their argument is based on biased measurement methodology. Previous studies that used the gold standard measure of individual salt intake, multiple non-consecutive 24-hour urine collections, to measure the salt exposure of their participants have consistently suggested that the optimal daily salt exposure is well below $6 \mathrm{~g} .{ }^{42}$

\section{Public health implications}

Our study confirms and quantifies the positive impact of the currently implemented UK salt reduction policies on CVD and GCa disease burdens. The overall health potential from salt reduction policies is likely to be greater, for example, through kidney disease, which we have not considered in our study. However, we also highlight two culprits of current policy. First, the national target of $6 \mathrm{~g} /$ day is unlikely to be reached in the next 15 years assuming the decline continues to be logarithmic. Second, the current policy will probably not reduce socioeconomic inequalities in CVD incidence and might even increase inequalities in GCa.

Structural policies, like a mandatory reformulation of processed foods, could potentially accelerate the decline in salt consumption and reduce absolute inequality in CVD. The existing salt reduction recommendations for the food industry could achieve the national target. ${ }^{9}$ In order to realise this, however, the food industry must comply with them, which is not happening at present. ${ }^{43}$ Failing to do so will most affect the poorest in society. Although we did not consider cost in our study, previous studies have suggested that mandatory reformulation is cost-effective and potentially cost-saving. ${ }^{44} 45$
Many experts are supporting now the combined reformulation in portion sizes, sugar, salt and fat content of processed food with sanctions for food manufacturers that do not comply. ${ }^{46}$ After the derail of the salt reduction strategy in 2011 due to the 'Responsibility Deal', that transferred the responsibility for nutrition from the Food Standards Agency to the food industry itself, salt reduction efforts have been renewed since $2014 .^{7}$ In fact, the second year of the Public Health England sugar reformulation programme is scheduled to also address salt in $2017 .{ }^{47}$

\section{Strengths and limitations}

Our study uses a technically advanced microsimulation model that synthesises information from the best available sources of information on population exposures to salt, and other non-communicable disease-related risk factor, to generate a 'close-to-reality' synthetic population. Many assumptions must be made with such models. Yet, in spite of the potential frailty of such assumptions, this model validated well against observed CVD and GCa incidence and mortality in real populations, even when multiply stratified. This validation is particularly important because for the years after 2006 the incidence and mortality in the synthetic population were recreated from first epidemiological principles and not through an optimisation process. Moreover, to ensure transparency, we have made IMPACT $\mathrm{NCD}_{\mathrm{NC}}$ source code open under GNU GPLv3 licence.

This study has many limitations, three of which are noteworthy. First, for the evaluation of current policy, we assumed that the decline in salt consumption observed since 2003 was fully attributable to the implemented policy. This was perhaps slightly simplistic and our estimates may, therefore, be high. Second, we did not model the effect of the 'Responsibility Deal' that potentially reduced the rate of salt decline since 2011. ${ }^{74}$ However, this overestimation of the baseline would, therefore, reduce the apparent gains from additional structural policies, making our conclusions relatively conservative. Third, we could not find a sufficiently large data set with individual-level 24-hour urine sodium measurements and other non-communicable disease-related risk factor information. The stochastic process we developed to overcome this and synthesise information from multiple sources increased the overall uncertainty of the model. Nevertheless, this uncertainty has been quantified and transparently reported using uncertainty intervals. 
Table 4 Additional effectiveness of structural policies compared with the 'current policy' scenario by quantile group of Index of Multiple Deprivation (QIMD)

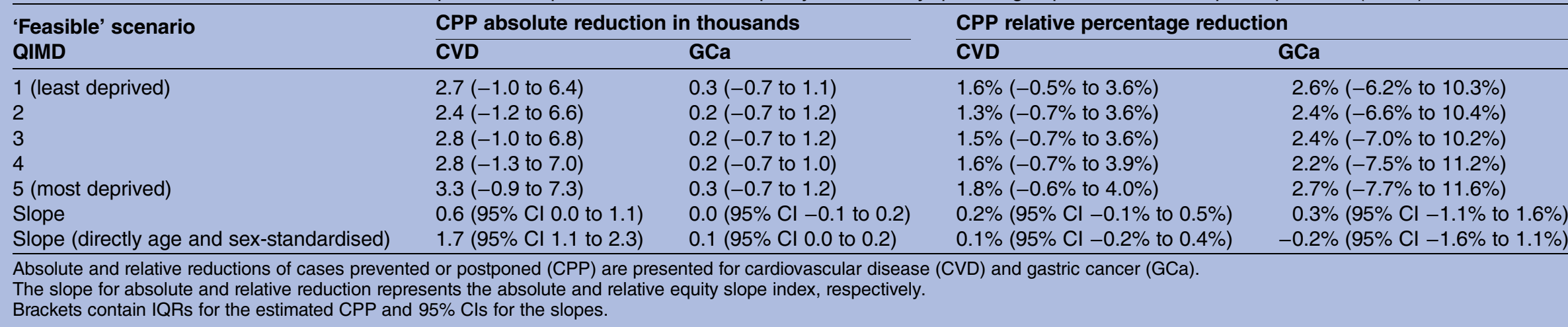

Table 5 The additional effectiveness of 'ideal' compared with the 'current policy' scenario by quantile group of Index of Multiple Deprivation (QIMD)

\begin{tabular}{|c|c|c|c|c|}
\hline \multirow{2}{*}{$\begin{array}{l}\text { ‘Ideal’ scenario } \\
\text { QIMD }\end{array}$} & \multicolumn{2}{|c|}{ CPP absolute reduction in thousands } & \multicolumn{2}{|c|}{ CPP relative percentage reduction } \\
\hline & $\overline{C V D}$ & GCa & $\overline{C V D}$ & GCa \\
\hline 1 (least deprived) & 7.7 (3.3 to 12.6$)$ & $0.8(-0.3$ to 1.7$)$ & $4.2 \%(2.0 \%$ to $6.5 \%)$ & $6.7 \%(-2.7 \%$ to $15.2 \%)$ \\
\hline 2 & 8.2 (3.6 to 12.6$)$ & $0.7(-0.2$ to 1.7$)$ & $4.1 \%(1.9 \%$ to $6.2 \%)$ & $5.6 \%(-1.7 \%$ to $14.4 \%)$ \\
\hline 3 & $8.9(4.0$ to 14.4$)$ & $1.0(-0.1$ to 2.0$)$ & $4.4 \%(2.1 \%$ to $6.9 \%)$ & $8.5 \%(-0.9 \%$ to $17.4 \%)$ \\
\hline 4 & 8.6 (3.5 to 13.3$)$ & $0.7(-0.2$ to 1.6$)$ & $4.4 \%(1.9 \%$ to $6.7 \%)$ & $6.8 \%(-2.0 \%$ to $15.8 \%)$ \\
\hline 5 (most deprived) & 9.7 (4.7 to 14.8$)$ & $1.0(0.1$ to 1.9$)$ & $4.9 \%(2.5 \%$ to $7.1 \%)$ & $9.3 \%(1.0 \%$ to $18.4 \%)$ \\
\hline Slope & $2.1(95 \% \mathrm{Cl} 1.4$ to 2.8$)$ & $0.3(95 \% \mathrm{Cl} 0.1$ to 0.4$)$ & $0.8 \%(95 \% \mathrm{Cl} 0.5 \%$ to $1.2 \%)$ & $3.4 \%(95 \% \mathrm{Cl} 2.0 \%$ to $4.7 \%)$ \\
\hline Slope (directly age and sex-standardised) & 5.7 (95\% Cl 5.0 to 6.3$)$ & $0.6(95 \% \mathrm{Cl} 0.4$ to 0.7$)$ & $0.7 \%(95 \% \mathrm{Cl} 0.3 \%$ to $1.0 \%)$ & $2.9 \%(95 \% \mathrm{Cl} 1.5 \%$ to $4.3 \%)$ \\
\hline
\end{tabular}




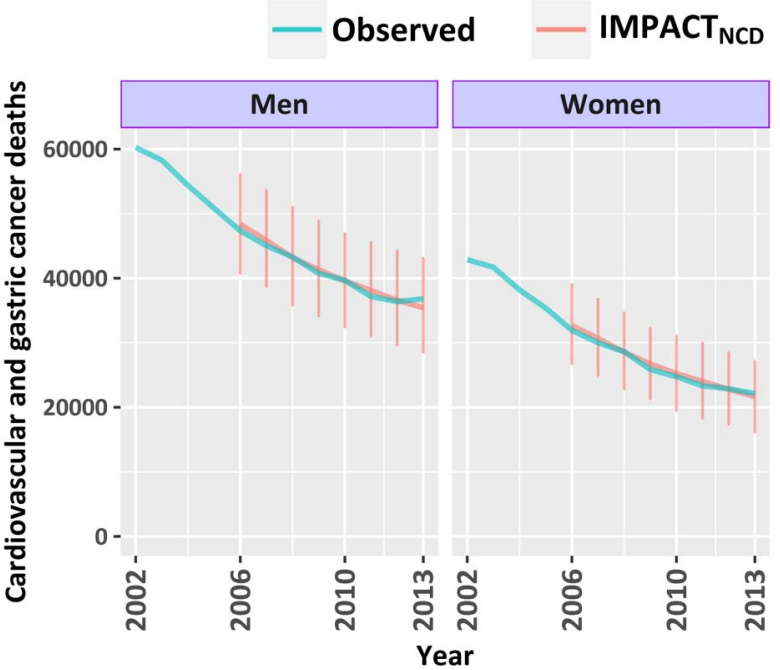

Figure 2 Number of deaths from cardiovascular disease and gastric cancer in England, by year and sex for ages 30-84. Office for National Statistics (ONS)-reported deaths (observed) versus IMPACT ${ }_{\text {NCD }}$-estimated. Observed deaths after 2010 were adjusted to account for changes in the ICD-10 version used by ONS since $2011 .{ }^{36}$ Error bars represent IQRs.

\section{CONCLUSIONS}

Current salt reduction policies are generally effective in reducing the cardiovascular and cancer disease burden but fail to do so equitably. Additional structural policies could achieve further, more equitable health benefits.

Contributors All authors made a substantial contribution to conception and design. CK, MG-C and MOF had the original idea. LH performed the literature search. CK prepared and conducted data analysis and modelling. All authors contributed to drafting the manuscript and revising it critically.

Funding The Health Survey for England was funded by the Department of Health until 2004 and the Health and Social Care Information Centre from 2005. IB and CK were supported by Medical Research Council Health eResearch Centre grant MR/K006665/1. SC, MOF, MGC, LH and PB were supported by the National Institute for Health Research through a grant (SPHR-LIL-PH1-MCD) to the LiLaC collaboration between the University of Liverpool and Lancaster University.

Disclaimer The funders had no role in study design, data collection and analysis, decision to publish or preparation of the manuscript.

Competing interests All authors have completed the ICMJE uniform disclosure form at http://www.icmje.org/coi_disclosure.pdf and declare: no support from any organisation for the submitted work; no financial relationships with any organisations that might have an interest in the submitted work in the previous 3 years, no other relationships or activities that could appear to have influenced the submitted work.

Ethics approval Ethical approval was not required for this study, as it is an analysis of previously collected data. Ethical approval for each survey was obtained by the Health Survey for England team.

Provenance and peer review Not commissioned; externally peer reviewed.

Data sharing statement Anonymised, non-identifiable participant-level cross-sectional survey data are freely available for academic researchers and public health staff to download from the UK Data Service. The source code of IMPACT $_{\text {NCD }}$ is available at https://github.com/ChristK/IMPACTncd/tree/ Evaluation_of_UK_salt_strategy.

Open Access This is an Open Access article distributed in accordance with the terms of the Creative Commons Attribution (CC BY 4.0) license, which permits others to distribute, remix, adapt and build upon this work, for commercial use, provided the original work is properly cited. See: http:// creativecommons.org/licenses/by/4.0/

\section{REFERENCES}

1. Strazzullo P, D'Elia L, Kandala NB, et al. Salt intake, stroke, and cardiovascular disease: meta-analysis of prospective studies. BMJ 2009;339:b4567.

2. D'Elia L, Rossi G, Ippolito R, et al. Habitual salt intake and risk of gastric cancer: a meta-analysis of prospective studies. Clin Nutr 2012;31:489-98.

3. Mozaffarian D, Fahimi S, Singh GM, et al. Global sodium consumption and death from cardiovascular causes. N Engl J Med 2014;371:624-34

4. Trieu K, Neal B, Hawkes C, et al. Salt reduction initiatives around the world - a systematic review of progress towards the global target. PLOS ONE 2015;10:e0130247.

5. Alwan A, Armstrong T, Bettcher D, et al. Global status report on noncommunicable diseases 2010. Geneva: World Health Organization, 2011. http://whqlibdoc.who.int/publications/2011/ 9789240686458_eng.pdf (accessed 14 Nov2016).

6. Webster JL, Dunford EK, Hawkes C, et al. Salt reduction initiatives around the world. J Hypertens 2011;29:1043-50.

7. MacGregor GA, He FJ, Pombo-Rodrigues S. Food and the responsibility deal: how the salt reduction strategy was derailed. $B M J$ 2015;350:h1936.

8. He FJ, Brinsden HC, MacGregor GA. Salt reduction in the United Kingdom: a successful experiment in public health. J Hum Hypertens 2014;28:345-52.

9. Wyness LA, Butriss JL, Stanner SA. Reducing the population's sodium intake: the UK Food Standards Agency's salt reduction programme. Public Health Nutr 2012;15:254-61.

10. Sadler K, Nicholson S, Steer T, et al. National diet and nutrition survey-assessment of dietary sodium in adults (aged 19 to 64 years) in England, 2011. London: Department of Health, 2012. http:// webarchive.nationalarchives.gov.uk/20130402145952/http:// transparency.dh.gov.uk/2012/06/21/sodium-levels-among-adults/ (accessed 14 Nov2016).

11. Scientific Advisory Committee on Nutrition. Salt and health. Norwich UK: The stationery office, 2003. https://www.gov.uk/government/ uploads/system/uploads/attachment_data/file/338782/SACN_Salt_ and_Health_report.pdf (accessed 14 Nov2016).

12. Ji C, Kandala NB, Cappuccio FP. Spatial variation of salt intake in Britain and association with socioeconomic status. BMJ Open 2013;3:e002246.

13. Ji C, Cappuccio FP. Socioeconomic inequality in salt intake in Britain 10 years after a national salt reduction programme. BMJ Open 2014;4:e005683.

14. McLaren L, Mclntyre L, Kirkpatrick S. Rose's population strategy of prevention need not increase social inequalities in health. Int $J$ Epidemiol 2010;39:372-7.

15. Lorenc T, Petticrew M, Welch V, et al. What types of interventions generate inequalities? Evidence from systematic reviews. $J$ Epidemiol Community Health 2013;67:190-3.

16. McGill R, Anwar E, Orton L, et al. Are interventions to promote healthy eating equally effective for all? Systematic review of socioeconomic inequalities in impact. BMC Public Health 2015;15:457.

17. Adams J, Mytton $\mathrm{O}$, White $\mathrm{M}$, et al. Why are some population interventions for diet and obesity more equitable and effective than others? The role of individual agency. PLOS Med 2016;13: e1001990.

18. Millett $\mathrm{C}$, Laverty AA, Stylianou $\mathrm{N}$, et al. Impacts of a national strategy to reduce population salt intake in England: serial cross sectional study. PLOS ONE 2012;7:e29836.

19. Cobiac LJ, Vos T, Veerman JL. Cost-effectiveness of interventions to reduce dietary salt intake. Heart 2010;96:1920-5.

20. Gillespie DO, Allen K, Guzman-Castillo M, et al. The health equity and effectiveness of policy options to reduce dietary salt intake in England: policy forecast. PLoS ONE 2015;10:e0127927.

21. Farr Institute. Investment in supercomputer accelerates the pace of health research across the North of England. http://www. farrinstitute.org/news/investment-in-supercomputer-accelerates-thepace-of-health-research-across-the-north-of-england (accessed 26 Apr 2016).

22. National Centre for Social Research, University College London. Department of Epidemiology and Public Health. Health Survey for England, 2001. 3rd edn. Colchester, Essex: UK Data Archive [distributor], 2010. (accessed 1 May 2014). 
23. Alfons A, Kraft S, Templ M, et al. Simulation of close-to-reality population data for household surveys with application to EU-SILC. Stat Methods Appl 2011;20:383-407.

24. National Centre for Social Research. An assessment of dietary sodium levels among adults (aged 19-64) in the UK general population in 2008 , based on analysis of dietary sodium in 24-hour urine samples. 2008. http://tna.europarchive.org/ 20110116113217/http://www.food.gov.uk/multimedia/pdfs/ 08sodiumreport.pdf

25. National Centre for Social Research. An assessment of dietary sodium levels among adults (aged 19-64) in the general population in England, based on analysis of dietary sodium in 24-hour urine samples. 2006. http://tna.europarchive.org/20110116113217/http:// www.food.gov.uk/multimedia/pdfs/englandsodiumreport.pdf (accessed 14 Nov 2016).

26. Henderson L, Irving K, Gregory J, et al. National Diet and Nutrition Survey: adults aged 19 to 64 years. Volume 3: Vitamin and mineral intake and urinary analytes. London: The Stationery Office, 2003. http://www.ons.gov.uk/ons/rel/lifestyles/the-national-diet-andnutrition-survey/2001-edition/the-national-diet-and-nutrition-survey--volume-3.pdf (accessed 5 Jun 2015).

27. Clark CE. Letter to the editor-the PERT model for the distribution of an activity time. Oper Res 1962;10:405-6.

28. World Cancer Research Fund International/American Institute for Cancer Research. Continuous Update Project report: diet, nutrition, physical activity and stomach cancer. AICR/WCRF. 2016. http://wcrf. org/sites/default/files/Stomach-Cancer-2016-Report.pdf (accessed 22 Apr 2016).

29. Department for Communities and Local Government. English indices of deprivation 2010-Publications-GOV.UK. 2011. https://www.gov. uk/government/statistics/english-indices-of-deprivation-2010 (accessed 26 Aug 2014)

30. Mackenbach JP, Kunst AE. Measuring the magnitude of socioeconomic inequalities in health: An overview of available measures illustrated with two examples from Europe. Soc Sci Med 1997;44:757-71.

31. Koerkamp B, Stijnen T, Weinstein MC, et al. The combined analysis of uncertainty and patient heterogeneity in medical decision models. Med Decis Making 2011;31:650-61.

32. Townsend N, Wickramasinghe K, Bhatnagar P, et al. Coronary heart disease statistics 2012 edition. British Heart Foundation, 2012. https:// http://www.bhf.org.uk/-/media/files/publications/ research/2012_chd_statistics_compendium.pdf (accessed 14 Nov 2016).

33. Stephens MR, Blackshaw GRJC, Lewis WG, et al. Influence of socio-economic deprivation on outcomes for patients diagnosed with gastric cancer. Scand J Gastroenterol 2005;40:1351-7.
34. Siemerink EJM, Hospers GAP, Mulder NH, et al. Disparities in survival of stomach cancer among different socioeconomic groups in North-East Netherlands. Cancer Epidemiol 2011;35:413-16.

35. Allemani $\mathrm{C}$, Weir HK, Carreira $\mathrm{H}$, et al. Global surveillance of cancer survival 1995-2009: analysis of individual data for 25676887 patients from 279 population-based registries in 67 countries (CONCORD-2). Lancet 2015;385:977-1010.

36. Office for National Statistics. Results of the ICD-10 v2010 bridge coding study, England and Wales. 2011. http://www.ons.gov.uk/ons/ rel/subnational-health3/results-of-the-icd-10-v2010-bridge-codingstudy-england-and-wales-2009/2009/index.html (accessed 21 Sep 2015)

37. Barton P, Andronis L, Briggs A, et al. Effectiveness and cost effectiveness of cardiovascular disease prevention in whole populations: modelling study. BMJ 2011;343:d4044.

38. Dodhia H, Phillips K, Zannou M-I, et al. Modelling the impact on avoidable cardiovascular disease burden and costs of interventions to lower SBP in the England population. J Hypertens 2012;30:217-26.

39. Hendriksen MA, van Raaij JMA, Geleijnse JM, et al. Health gain by salt reduction in Europe: a modelling study. PLOS ONE 2015;10: e0118873.

40. Mente A, O'Donnell M, Rangarajan S, et al. Associations of urinary sodium excretion with cardiovascular events in individuals with and without hypertension: a pooled analysis of data from four studies. Lancet 2016;388:465-75.

41. O'Donnell M, Mente A, Rangarajan S, et al. Urinary sodium and potassium excretion, mortality, and cardiovascular events. $N$ Engl J Med 2014;371:612-23.

42. Cogswell ME, Mugavero $\mathrm{K}$, Bowman BA, et al. Dietary sodium and cardiovascular disease risk-measurement matters. N Engl J Med 2016;375:580-6.

43. Knai C, Petticrew M, Durand MA, et al. Has a public-private partnership resulted in action on healthier diets in England? an analysis of the public health responsibility deal food pledges. Food Policy 2015;54:1-10.

44. Collins M, Mason H, O'Flaherty M, et al. An economic evaluation of salt reduction policies to reduce coronary heart disease in England: a policy modeling study. Value Health 2014;17:517-24

45. Mason H, Shoaibi A, Ghandour R, et al. A cost effectiveness analysis of salt reduction policies to reduce coronary heart disease in four Eastern Mediterranean countries. PLOS ONE 2014;9:e84445.

46. Obesity Health Alliance. Reformulation briefing. 2016. http:// obesityhealthalliance.org.uk/wp-content/uploads/2016/08/ Reformulation-briefing-FINAL.pdf (accessed 11 Dec 2016).

47. HM Government. Childhood obesity: a plan for action. 2016. https:// www.gov.uk/government/publications/childhood-obesity-a-plan-foraction (accessed 11 Dec 2016). 